\title{
Changes in antioxidant and probiotic properties of a freeze-dried apple snack during storage
}

\section{Burca, C. ${ }^{\text {; }}$ Seguí, L. ${ }^{\text {a }}$ Betoret, N. ${ }^{\text {; }}$ Barrera, C. ${ }^{\mathrm{a}}$}

Functional Foods group. Institute of Food Engineering for Development. Universitat Politècnica de València, Valencia, Spain

*E-mail of the corresponding author: noebeva@tal.upv.es

\begin{abstract}
This research developed an apple snack with potential probiotic effect $\left(>10^{7}\right.$ CFU/g) by combining vacuum impregnation with Lactobacillus salivarius spp. salivarius (CECT 4063) and freeze-drying. Throughout storage (30 days), both the lactobacillus viability and the total flavonoids content decreased. Trehalose adition (10\% by weight) to the impregnation liquid and/or its homogenization at $100 \mathrm{MPa}$ accelerated the loss of cell viability but delayed flavonoids degradation and promoted an increase in the amount of phenols and total antioxidants.
\end{abstract}

Keywords: L. salivarius spp. salivarius; homogenization; trehalose; freezedrying; antioxidants. 


\section{Introduction}

Helicobacter pylori is a pathogenic bacterium that causes severe gastric problems to a large part of the world's population, especially in less developed countries [1]. Traditional treatments based on antibiotics have side effects and are not $100 \%$ effective [2]. Recent studies show that some strains of the Lactobacillus genus are effective in the treatment against Helicobacter pylori, reducing the colonization of this pathogen, what has promoted the incorporation of Lactobacillus in the formulation of certain foods [3]. However, the survival of these microorganisms in food is rather limited, especially in not dairy products. Some food engineering techniques, such as the formulation with certain ingredients (e.g. probiotics) or the modification of the structures conferring protection to the microorganism (e.g. encapsulation) can be applied in order to alter probiotics functionality and/or increase their survival against adverse conditions. Specifically, in this study the effect of trehalose addition (10\%, w/w) to the impregnation liquid and/or its homogenization at $100 \mathrm{MPa}$ on Lactobacillus salivarius spp. salivarius (CECT 4063) survival during the manufacture and storage of a freeze-dried apple snack was evaluated. Given the high content in antioxidant compunds of the raw materials, also changes in this bioactive substances were analyzed.

\section{Materials and Methods}

\subsection{Solid matrix}

Apples (var. Granny Smith) cut into $5 \mathrm{~mm}$ thick rings (20 and $65 \mathrm{~mm}$ of internal and external diameter, respectively) were used as solid matrix for the snack preparation.

\subsection{Impregnation liquids}

The impregnation liquid was prepared from commercial clementine juice (Hacendado brand). Following the procedure described by Betoret et al. [4], yeast extract (5 g/L) and sodium bicarbonate (9,8 g/L) were added for the optimal microbial growth. When required, $100 \mathrm{~g} / \mathrm{kg}$ of food grade trehalose (TREHA ${ }^{\mathrm{TM}}$, Cargill, Barcelona, Sapin) were also added to the juice formulation. Once all the ingredients were dissolved, the liquids were inoculated $\left(10^{9} \mathrm{CFU} / \mathrm{L}\right)$ with strain CECT 4063 of Lactobacillus salivarius spp. salivarius (Colección Española de Cultivos Tipo, Universitat de València, Burjassot, Spain) that had been previously grown on MRS Broth agar. After $24 \mathrm{~h}$ of incubation at $37^{\circ} \mathrm{C}$, part of the liquids were homogenized at $100 \mathrm{MPa}$ on a laboratory scale high pressure homogenizer (Panda Plus 2000, GEA-Niro Soavi, Parma, Italy) before their use as impregnation liquids. Four different impregnation liquids were prepared in total (Table 1).

Table 1. Different impregnation liquids employed in the present study.

\begin{tabular}{c|cc}
\hline Impregation liquid & Trehalose (g/kg) & Pressure (MPa) \\
\hline $0 \% \_0 \mathrm{MPa}$ & 0 & 0 \\
$0 \% \_100 \mathrm{MPa}$ & 0 & 100 \\
$10 \% \_0 \mathrm{MPa}$ & 100 & 0 \\
$10 \% \_100 \mathrm{MPa}$ & 100 & 100 \\
\hline
\end{tabular}




\subsection{Experimental procedure}

This section describes the unit operations involved in the manufacture of apple snacks enriched with Lactobacillus salivarius spp. salivarius (CECT 4063).

First, vacuum impregnation was performed in a vacuum chamber (HERAEUS Vacuun Oven, THERMO SCIENTIFIC) connected to a vacuum pump (ILMVAC, Germany). A vacuum pressure of 50 mbar was applied for 10 min to the apple rings immersed in the corresponding liquid. Then, the atmospheric pressure was restored and maintained for another $10 \mathrm{~min}$.

Vacuum impregnated apple rings were deep-frozen and kept at $-40{ }^{\circ} \mathrm{C}$ for $24 \mathrm{~h}$ (Matek model CVN-40/105). Then, they were placed in a pilot scale freeze-drier (TELSTAR LIOALFA 680) at $-45^{\circ} \mathrm{C}$ and a vacuum pressure of 0.1 mbar for $24 \mathrm{~h}$ more.

In the end, freeze-dried apple slices were stored in hermetic and opaque bags and kept under controlled conditions of humidity and temperature for 30 days.

\subsection{Analytical determinations}

All the analytical determinations were carried out at least in triplicate on liquid and/or solid samples at different stages throughout the snack manufacture process.

\subsubsection{Water content and water activity}

The apple samples moisture content was determined by drying a known amount of sample in a vacumm oven at $60^{\circ} \mathrm{C}$ and 200 mbar until it reached a constant weight.

The water activity of apple samples was measured at $25^{\circ} \mathrm{C}$ in a properly calibrated dew poin hygrometer (Decagon Aqualab model CX-2, with an accuracy of \pm 0.003 ).

\subsubsection{Antioxidant properties}

Extracts from solid samples were obtained by mixing $2 \mathrm{~g}$ of fresh and impregnated apple or $0.35 \mathrm{~g}$ of freeze-dried apple with $10 \mathrm{~mL}$ of a 80:20 (v/v) methanol-water solution.

Total phenols content was measured at $760 \mathrm{~nm}$ in a Helios Zeta UV/Vis Thermo Scientific spectrophotometer by the Folin-Ciocalteu reagent method [5]. Results were expressed in milligrams of gallic acid equivalents per gram of sample (mg GAE/g).

Total flavonoids content was measured at $368 \mathrm{~nm}$ in a Helios Zeta UV/Vis Thermo Scientific spectrophotometer by the colorimetric method of aluminum chloride [6]. Results were expressed in milligrams of quercetin equivalents per gram of sample (mg QE/g).

Antioxidant activity was determined at $515 \mathrm{~nm}$ in a Helios Zeta UV/Vis Thermo Scientific spectrophotometer by the DPPH method [7]. Results were expressed in milligrams of trolox equivalents per gram of sample (mg TE/g). 


\subsubsection{Viable counts}

The Lactobacillus salivarius spp. salivarius (CECT 4063) concentration was analyzed both in the growing medium, the impregnation liquids and the apple samples by the serial dilution and plating method. MRS seeded plates were incubated in anaerobiosis at $37^{\circ} \mathrm{C}$ for 3 days. In the case of solid apple samples, the first dilution was carried out in a stomacher bag in which $5 \mathrm{~g}$ of sample were crushed with $45 \mathrm{~mL}$ of sterile peptone water.

\subsection{Statistical analysis}

The effect that the different variables considered exert on the obtained results was evaluated with the Statgraphics Centurion XVI program by simple analysis (simple ANOVA) with a 95\% confidence level.

\section{Results and discussion}

\subsection{Changes in Lactobacillus salivarius spp. salivarius (CECT 4063) content}

As it is shown in Fig. 1, Lactobacillus salivarius spp. salivarius (CECT 4063) content in the impregnation liquid (VI liquid) increased significantly by adding $10 \%$ of trehalose by weight to its formulation or by its homogenization at $100 \mathrm{MPa}$. However, the combination of both factors did not notably improve the viable counts.

Similar trends were observed in vacuum impregnated apples (VI apple), whose microbial content was significantly lower than that of any of the impregnation liquids. This is logical considering that only $20 \%$ of fresh apple volume is filled with the impregnation liquid during the vacuum impregnation step [8].

Finally, freeze-dried apple samples (FD apple) potential probiotic effect was higher than that of vacuum impregnated ones, but not as high as expected from the decrease in their water content (from $85.3 \pm 1.2 \%$ in VI apple to $4.97 \pm 1.02 \%$ in FD apple). Regarding the composition of the impregnation liquid, the addition of trehalose to its composition slightly reduced the adverse effect of freeze-drying on the microbial population. On the contrary, the subsequent homogenization of trehalose enriched juice increased $L$. salivarius spp. salivarius vulnerability to the freeze-drying step. 


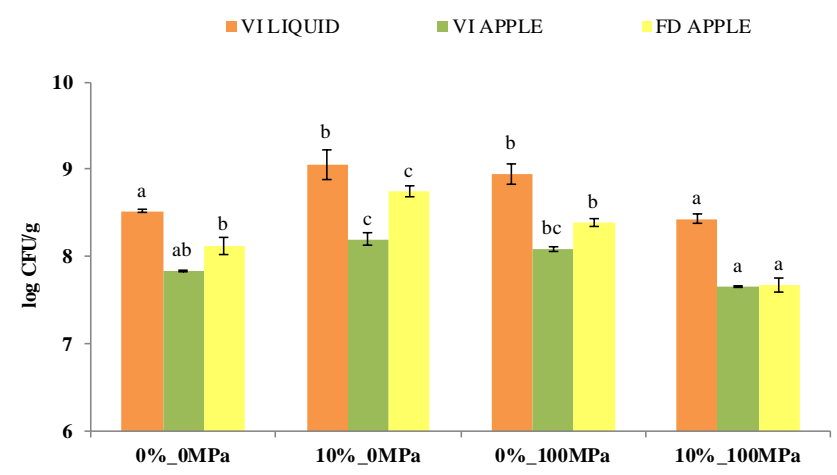

Fig. 1. Microbial counts in the impregnation liquids and both in vacuum impregnated (VI) and freeze-dried (FD) apple samples. Different letters within a single series indicate statistical significant differences at $95 \%$ confidence level.

Throughout storage (Fig. 2), L. salivarius spp. salivarius (CECT 4063) content in freezedried apple samples suffered a notable decline, in spite of the low water activity reached by the snack $(0.27 \pm 0.02)$. This fact was particularly evident when trehalose and/or pressure were applied to the impregnating liquid, thus suggesting that the stress caused by the osmotic and/or the pressure gradient favored the loss of vialbility and the shortening of the snack self life. Just to mention that for a food to be considered probiotic it must contain at least $10^{7}$ CFU/g when consumed [9]. Only snacks impregnated with liquids 0\%_0MPa and $10 \%$ 0MPa met this condition at the end of the storage.

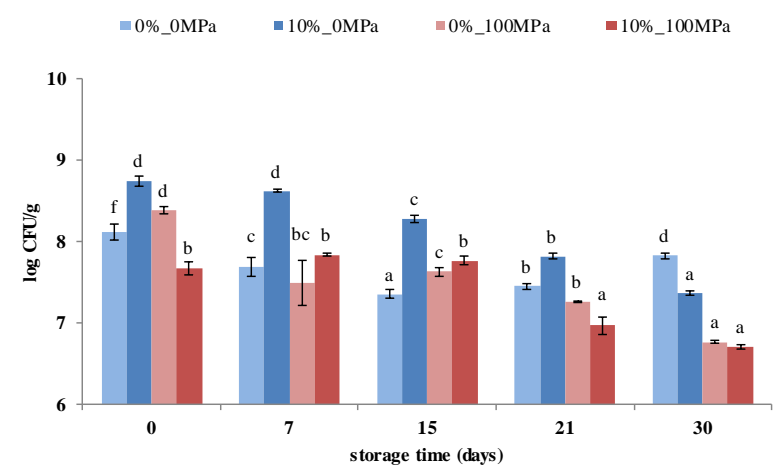

Fig. 2. Changes in microbial counts troughout storage of freeze-dried apple snacks. Different letters within a single series indicate statistical significant differences at $95 \%$ confidence level. 


\subsection{Changes in antioxidant properties}

Despite the notable differences observed in their total phenols and flavonoids content, antioxidant capacity assessed by DPPH method both in the impregnation liquids and the vacuum impregnated apple samples were of the same order (Table 2). As expected, all the antioxidants increased their concentration significantly after the freeze-drying step. As for the composition of the vaccum impregnation liquid, neither the addition of trehalose nor the homogenization resulted in a final snack with improved antioxidant properties.

Table 2. Antioxidant properties of the impregnation liquids and both the VI and FD apple samples.

\begin{tabular}{|c|c|c|c|c|}
\hline $\begin{array}{c}\text { Food } \\
\text { matrix }\end{array}$ & $\begin{array}{l}\text { Impregation } \\
\text { liquid }\end{array}$ & $\begin{array}{l}\text { total phenols } \\
\text { (mg GAE/g) }\end{array}$ & $\begin{array}{l}\text { total flavonoids } \\
\text { (mg QE/g) }\end{array}$ & $\begin{array}{c}\text { DPPH } \\
\text { (mg TE/g) }\end{array}$ \\
\hline \multirow{4}{*}{ VI liquid } & 0\%_0MPa & $0.78(0.07)^{\mathrm{a}}$ & $1.01(0.07)^{\mathrm{c}}$ & $0.70(0.04)^{\mathrm{a}}$ \\
\hline & 10\%_0МРa & $0.79(0.08)^{\mathrm{a}}$ & $0.904(0.004)^{\mathrm{c}}$ & $0.78(0.06)^{\mathrm{a}}$ \\
\hline & 0\%_100 MPa & $0.7235(0.0007)^{\mathrm{a}}$ & $0.954(0.014)^{\mathrm{c}}$ & $0.7(0.2)^{\mathrm{a}}$ \\
\hline & $10 \% \_100 \mathrm{MPa}$ & $0.82(0.03)^{\mathrm{a}}$ & $0.93(0.03)^{c}$ & $0.73(0.05)^{\mathrm{a}}$ \\
\hline \multirow{4}{*}{ VI apple } & 0\%_0MPa & $0.441(0.015)^{\mathrm{a}}$ & $0.291(0.002)^{b}$ & $0.89(0.12)^{\mathrm{a}}$ \\
\hline & 10\%_0MPa & $0.34(0.06)^{\mathrm{a}}$ & $0.175(0.003)^{\mathrm{a}}$ & $0.61(0.03)^{\mathrm{a}}$ \\
\hline & 0\%_100 MPa & $0.82(0.11)^{\mathrm{a}}$ & $0.217(0.002)^{\mathrm{ab}}$ & $0.92(0.07)^{\mathrm{a}}$ \\
\hline & 10\%_100 MPa & $0.64(0.03)^{\mathrm{a}}$ & $0.132(0.002)^{\mathrm{a}}$ & $0.77(0.04)^{\mathrm{a}}$ \\
\hline \multirow{4}{*}{ FD apple } & 0\%_0MPa & $11.4(0.6)^{\mathrm{e}}$ & $8.70(0.07)^{\mathrm{f}}$ & $7.84(0.07)^{\mathrm{d}}$ \\
\hline & 10\%_0MPa & $3.025(0.012)^{b}$ & $1.186(0.012)^{\mathrm{d}}$ & $5.5(0.2)^{c}$ \\
\hline & 0\%_100 MPa & $6.0(0.3)^{\mathrm{d}}$ & $2.01(0.14)^{\mathrm{e}}$ & $5.0(0.3)^{c}$ \\
\hline & 10\%_100 MPa & $4.8(0.4)^{\mathrm{c}}$ & $1.19(0.02)^{\mathrm{d}}$ & $2.3(0.8)^{\mathrm{b}}$ \\
\hline
\end{tabular}

Mean values and standard deviation in brackets. Different superscripts in the same column indicate statistical significant differences at 95\% confidence level.

Regarding the stability of the antioxidant compounds throughout the snack storage, it is shown in Fig. 3 the change in each component concentration from the beginning to the end of the storage referred to the initial concentration $\left(\Delta \mathrm{x}^{\mathrm{i}}\right)$ :

$$
\Delta \mathrm{x}^{\mathrm{i}}(\%)=\frac{\mathrm{x}_{\mathrm{t}=0}^{\mathrm{i}}-\mathrm{x}_{\mathrm{t}=30}^{\mathrm{i}}}{\mathrm{x}_{\mathrm{t}=0}^{\mathrm{i}}} \cdot 100
$$

where $\mathrm{x}_{\mathrm{t}=0}^{\mathrm{i}}$ and $\mathrm{x}_{\mathrm{t}=30}^{\mathrm{i}}$ indicate the component $\mathrm{i}$ concentration in freshly made and 30 days stored FD apples, respectively (g of component $\mathrm{i} /$ total g).

Generally speaking, the total phenols gain was significantly higher and the total flavonoids loss was significantly lower (p-value $<0.05$ ) in apple snacks that were impregnated with any of the liquids that included trehalose in its composition, but especially in those impregnated with the liquid that was additionally homogenized $\left(10 \% \_100 \mathrm{MPa}\right)$. As a result, these samples also showed a significantly higher increase in the total antioxidant content measured by the DPPH method. 


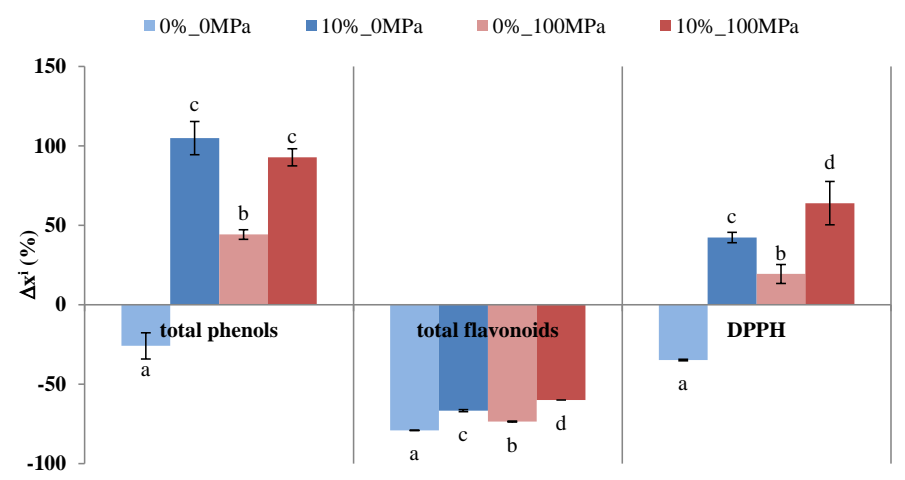

Fig. 3. Changes in antioxidant properties after 30 days of storage of freeze-dried apple snacks. Different letters within a single compound indicate statistical significant differences at $95 \%$ confidence level.

Given the ability of trehalose to protect biological structures [10], apple tissue would have been less damaged during the freeze-drying step. As a result, antioxidant compounds would have been less accessible to adverse conditions that promote their degradation. Trehalose protective effect could be promoted by the reduction in the particle size that implies the application of a homogenization step [11], thus favouring the inflow of a greater amount of liquid (and trehalose) into the apple porous structure during the vacuum impregnation.

\section{Conclussions}

Vacuum impregnation allows to incorporate lactobacillus into the apple porous structure to a greater or lesser extent, depending on the viable counts in the impregnation liquid. The subsequent freeze-drying increases apples stability without negatively affecting the microbial content or the antioxidants content. Lactobacillus salivarius spp. salivarius survival during the further storage was negatively affected by the addition of trehalose to the impregnation liquid and/or its homogenization. On the contrary, the addition of trehalose to the impregnation liquid and/or its homogenization delayed flavonoids degradation and promoted an increase in the amount of both phenols and total antioxidants.

\section{References}

[1] Taylor, D.E.; Blaser, M. The epidemiology of Helicobacter pylori infection. Epidemiologic Reviews 1991, 13: 42-59.

[2] Lopes, D.; Nunes, C.; Martins, M.C.; Sarmento, B.; Reis, S. Eradication of Helicobacter pylori: past, present and future. Journal of Controlled Release 2014, 189: 169-186.

[3] Hamilton-Miller, J.M. The role of probiotics in the treatment and prevention of Helicobacter pylori infection. International Journal of Antimicrobial Agents 2003, 22: 
360-366.

[4] Betoret, E.; Betoret, N.; Arilla, A.; Bennár, M.; Barrera, C.; Codoñer, P.; Fito, P. No invasive methodology to produce a probiotic low humid apple snack with potential effect against Helicobacter pylori. Journal of Food Engineering 2012, 110 (2), 289-293.

[5] Singleton, V.L.; Rossi, J.A. Colorimetry of total phenolics with phosphomolybdicphosphotungstic acid reagents. American Journal of Enology and Viticulture 1965, 16, 144-158.

[6] Luximon-Ramma, A.; Bahorun, T.; Crozier, A.; Zbarsky, V.; Datla, K.; Dexter, D.; Aruoma, O. Characterization of the antioxidant functions of flavonoids and proanthocyanicins in Muritian black teas. Food Research International 2005, 38, 357367.

[7] Brand-Williams, W.; Cuvelier, M.; Berset, C. Use of a free radical method to evaluate antioxidant activity. LWT-Food Science Technology 1995, 28 (1): 25-30.

[8] Fito, P.; Chiralt, A.; Barat, J.M.; Andrés, A.; Martínez-Monzó, J.; Martínez-Navarrete, $\mathrm{N}$. Vacuum impregnation for development of new dehydrated products. Journal of Food Engineering 2001, 49 (4): 297-302.

[9] Ranadheera, R.D.C.S.; Baines, S.K.; Adams, M.C. Importance of food in probiotic efficacy. Food Research International 2010, 43:1-7.

[10] Conrad, P.B.; Miller, D.P.; Cielenski, P.R.; De Pablo, J.J. Stabilization and preservation of Lactobacillus acidophilus in saccharide matrices. Cryobiology 2000, 41 (1): 17-24.

[11] Betoret, E.; Betoret, N.; Carbonell, J.V.; Fito, P. Effects of pressure homogenization on particle size and the functional properties of citrus juices. Journal of Food Engineering 2009, 92 (1): 18-23. 